\title{
Manejo e Prevenção de Reações Adversas da Quimioterapia Antineoplásica com Platinas em Pacientes com Cânceres Esofágico e Gástrico: Revisão Sistemática da Literatura
}

https://doi.org/10.32635/2176-9745.RBC.2021v67n4.1347

\author{
Management and Prevention of Adverse Reactions to Platinum Antineoplastic Chemotherapy in Patients with Esophageal \\ and Gastric Cancer: Systematic Literature Review \\ Manejo y Prevención de Reacciones Adversas a la Quimioterapia Antineoplásica con Platino en Pacientes con Cánceres \\ de Esófago y Gástrico: Revisión Sistemática de la Literatura
}

Ney Moura Lemos Pereira'; Telma Maria Araújo Moura Lemos²; Rand Randall Martins ${ }^{3}$; Roberto Fernandes da Costa ${ }^{4}$; Fernanda Nervo Raffin 5

RESUMO

Introdução: $\mathrm{O}$ câncer gástrico é a quinta doença maligna mais comum em todo o mundo. Trata-se do tumor maligno mais incidente na Ásia, especialmente na China. O carcinoma esofágico é um dos tipos mais agressivos de tumor maligno. Os tratamentos multimodais, incluindo quimioterapia neoadjuvante e quimiorradioterapia, são utilizados e podem causar fadiga, vômito, diarreia, alteraçôes cutâneas, caquexia e neuropatia periférica, que podem ser efeitos colaterais importantes para muitos pacientes que realizam seus tratamentos. Objetivo: Realizar uma revisão sistemática sobre o manejo e a prevenção de reaçôes adversas da quimioterapia antineoplásica com platinas em pacientes com câncer esofágico e tumor gástrico. Método: Para seleção dos artigos, foi realizada a busca em três bases de dados: MEDLINE/PubMed, Cochrane e Embase, com a estratégia PICO, variando os descritores MeSH/DeCs e operadores booleanos. Resultados: Foram encontrados 455 títulos, dos quais, após utilizar a diretriz PRISMA, restaram 15 artigos para a revisão sistemática, que abordavam o manejo e a prevenção de náusea e vômitos, neuropatia periférica, caquexia, suplementação de magnésio, tratamento de depressão e toxicidade geral. Conclusão: Verificou-se que náuseas, vômitos, neuropatia e hipomagnesemia tiveram maior número de estudos relacionados ao manejo e à prevenção desses sintomas, nos quais identificaram-se algumas sugestôes de condutas com maior evidência para essas reaçôes. As demais reaçôes encontradas ainda carecem de mais estudos, principalmente nos casos de cânceres gástrico e esofágico.

Palavras-chave: Neoplasias Gástricas; Neoplasias Esofágicas; Efeitos Colaterais e Reaçôes Adversas Relacionados a Medicamentos; Compostos de Platina; Antineoplásicos.

\section{ABSTRACT}

Introduction: Gastric cancer is the fifth most common malignancy worldwide. It is the most frequent malignant tumor in Asia, especially in China. Esophageal carcinoma is one of the more aggressive types of malignant tumor. Multimodal treatments, including neoadjuvant chemotherapy and chemoradiotherapy are utilized and can cause fatigue, vomiting, diarrhea, skin changes, cachexia, and peripheral neuropathy, which can be important side effects for many patients undergoing their treatments. Objective: Carry out a systematic review on the management and prevention of adverse reactions of antineoplastic chemotherapy with platinum in patients with esophageal cancer and gastric tumor. Method: To select the articles, a search was conducted in three databases: MEDLINE/PubMed, Cochrane and Embase, with the PICO strategy, alternating between $\mathrm{MeSH} / \mathrm{DeCs}$ descriptors and Boolean operators. Results: 455 titles were found, of which, after using the PRISMA guideline, 15 articles remained for systematic review, addressing the management and prevention of nausea and vomiting, peripheral neuropathy, cachexia, magnesium supplementation, treatment of depression and general toxicity. Conclusion: The greatest number of studies addressing the management and prevention of the symptoms of nausea, vomits, neuropathy and hypomagnesemia were found, and it was possible to identify some suggestions of conducts to treat these reactions. More studies are necessary for the other reactions encountered, mainly in the cases of gastric and esophageal cancer. Key words: Stomach Neoplasms; Esophageal Neoplasms; Drug-Related Side Effects and Adverse Reactions; Platinum Compounds; Antineoplastic Agents.

\section{RESUMEN}

Introducción: El cáncer gástrico es la quinta neoplasia maligna más común en todo el mundo. Es el tumor maligno más común en Asia, especialmente en China. El carcinoma de esófago es uno de los tipos de tumores malignos más agresivos. Se utilizan tratamientos multimodales, que incluyen quimioterapia neoadyuvante y quimiorradioterapia que pueden provocar: fatiga, vómitos, diarrea, alteraciones cutáneas, caquexia y neuropatía periférica, que pueden ser efectos secundarios importantes para muchos pacientes sometidos a sus tratamientos. Objetivo: Realizar una revisión sistemática sobre el manejo y prevención de reacciones adversas de la quimioterapia antineoplásica con platino en pacientes con cáncer de esófago y tumor gástrico. Método: Para la selección de los artículos se realizó una búsqueda en tres bases de datos: MEDLINE/PubMed, Cochrane y Embase, con la estrategia PICO, variando los descriptores $\mathrm{MeSH} / \mathrm{DeCs}$ y operadores booleanos. Resultados: Se encontraron 455 títulos, de los cuales, luego de utilizar la guía PRISMA, quedaron 15 artículos para revisión sistemática, que abordaron el manejo y prevención de náuseas y vómitos, neuropatía periférica, caquexia, suplementación con magnesio, tratamiento de la depresión y toxicidad general. Conclusión: Se verifico que náuseas, vómitos, neuropatía e hipomagnesemia tuvieron un mayor número de estudios relacionados con el manejo y prevención de los síntomas, en los cuales fue posible identificar algunas sugerencias de conducta con mayor evidencia de estas reacciones. Las otras reacciones encontradas aún necesitan más estudios, especialmente en casos de cánceres gástrico y de esófago.

Palabras clave: Neoplasias Gástricas; Neoplasias Esofágicas; Efectos Colaterales y Reacciones Adversas Relacionados con Medicamentos; Compuestos de Platino; Antineoplásicos.

1-5Universidade Federal do Rio grande do Norte (UFRN). Natal (RN), Brasil.

'E-mail: neymoura@yahoo.com. Orcid iD: https://orcid.org/0000-0003-1792-3672

2E-mail: telmaml@yahoo.com.br. Orcid iD: https://orcid.org/0000-0001-7118-2145

${ }^{3}$ E-mail: randrandall@gmail.com. Orcid iD: https://orcid.org/0000-0001-9668-0482

${ }^{4}$ E-mail: roberto@robertocosta.com.br. Orcid iD: https://orcid.org/0000-0002-8789-1744

5E-mail: feraffin@ufrnet.br. Orcid iD: https://orcid.org/0000-0001-7623-4485

Endereço para correspondência: Ney Moura Lemos Pereira. UFRN, Centro de Ciências da Saúde, Faculdade de Farmácia. Av. General Gustavo Cordeiro de Farias, S/N - Petrópolis. Natal (RN), Brasil. CEP 59012-570.E-mail: neymoura@yahoo.com 


\section{INTRODUÇÃO}

O câncer gástrico é a quinta doença maligna mais comum em todo o mundo. Trata-se do tumor maligno mais incidente na Ásia, especialmente na China. A maioria dos casos é diagnosticada como câncer gástrico avançado na admissão. A ressecção cirúrgica complementada com quimioterapia pós-operatória continua sendo o tratamento primário, enquanto a taxa de recorrência pós-operatória é alarmantemente alta ${ }^{1}$. O carcinoma esofágico é um dos tipos mais agressivos de tumor maligno. Os tratamentos multimodais, incluindo quimioterapia neoadjuvante e quimiorradioterapia, demonstraram melhorar a taxa de sobrevivência de pacientes com carcinoma esofágico localmente avançado ${ }^{2}$.

O tratamento sistêmico do câncer de esôfago metastático avançado, da junção gastroesofágica (GEJ) e do câncer gástrico utiliza uma combinação de múltiplos agentes quimioterápicos citotóxicos, embora não exista nenhum regime de tratamento padrão. A cisplatina e os agentes de platina estão entre os grupos de fármacos citotóxicos mais amplamente utilizados e bem-sucedidos em todo o mundo. A cada ano, mais de 5,8 milhóes de pacientes são diagnosticados com câncer, para os quais, a terapia de primeira linha potencialmente inclui agentes de platina. A adiçáo de cisplatina em altas doses à radioterapia exacerba os eventos adversos associados à radioterapia e causa alguns episódios específicos associados à cisplatina, que são dependentes da dose. Náuseas, vômitos, ototoxicidade, nefrotoxicidade e neurotoxicidade também são observados com o tratamento com cisplatina (monoterapia ou em combinação). Além disso, essas toxicidades são cumulativas, dependentes da dose, muitas vezes não reversíveis (exceto náuseas e vômitos) e podem envolver lesôes extensas em órgãos em regeneração, ou que não se regeneram, podendo impactar na qualidade de vida $(\mathrm{QV})$ de pacientes curados ${ }^{3-5}$.

No Brasil, segundo as estimativas do Instituto Nacional de Câncer José Alencar Gomes da Silva (INCA) ${ }^{6}$, o câncer de esôfago é sexto mais frequente entre os homens e o $15^{\circ}$ entre as mulheres, e o oitavo mais incidente no mundo. $\mathrm{O}$ câncer gástrico é o terceiro tipo mais comum entre homens, atingindo por volta dos 60-70 anos e o quinto entre as mulheres. Dessa maneira, protocolos para prevençáo e manejo para reaçóes adversas a medicamentos (RAM) dos derivados da platina são essenciais, representando um importante objeto de pesquisa. Destacam-se as diretrizes para cuidados de suporte do National Comprehensive Cancer Network $(\mathrm{NCCN})^{7}$, que abordam o assunto; porém, até onde se sabe, inexistem revisóes sistemáticas que consolidem o conhecimento acerca do manejo e da prevençâo da neuropatia, náusea, vômito, nefrotoxicidade, entre outras reaçóes adversas, em se tratando de doenças específicas como os cânceres de esôfago e gástrico.

Dessa forma, considerando essa lacuna no conhecimento, o objetivo deste trabalho foi revisar a literatura sobre o manejo e a prevençáo de reações adversas da quimioterapia antineoplásica com platinas em pacientes com cânceres esofágico e gástrico.

\section{MÉTODO}

Trata-se de uma revisão sistemática da literatura conduzida de acordo com o Preferred Reporting Items for Systematic reviews and Meta-Analyses (PRISMA) ${ }^{8}$. $\mathrm{O}$ estudo foi protocolado no International Prospective Register of Systematic Reviews (PROSPERO) sob o número CRD42020210705.

Foram incluídos estudos com pacientes com câncer gástrico, câncer de esôfago ou câncer gastroesofágico (usando qualquer critério de diagnóstico reconhecido). Os estudos, abordando vários tipos de câncer que especificassem a intervenção (manejo ou prevenção), mas não especificassem a doença, foram excluídos.

A busca foi realizada por dois revisores separadamente, selecionando os artigos primeiramente pela leitura dos títulos e resumos, seguindo os critérios de inclusão e exclusão. Em um segundo momento, procedeu-se à leitura completa do artigo fazendo-se a seleçáo de acordo com os critérios de elegibilidade. A revisão contou apenas com estudos observacionais e ensaios clínicos, não sendo utilizada literatura cinzenta. A elegibilidade dos artigos foi avaliada independentemente por dois revisores e as divergências foram resolvidas em conjunto por todos os autores. A pesquisa foi realizada nas bases de dados MEDLINE/PubMed, Embase e Cochrane Library, abordando câncer gástrico e câncer de esôfago, utilizando derivados de platinas e toxicidades sistêmicas. Em cada base de dados, foi realizada a pesquisa em sua totalidade, sendo elegíveis estudos dos últimos cinco anos, sem restrição de idioma. As pesquisas foram realizadas nos meses de julho e agosto de 2020. Utilizando-se das bases de dados anteriormente citadas e a estratégia $\mathrm{PICO}^{9}$, variando os descritores $\mathrm{MeSH} / \mathrm{DeCs}$ e operadores booleanos, selecionaram-se os artigos para aplicar a diretriz do PRISMA.

Usando formulário padronizado, os mesmos dois revisores, de forma independente, realizaram a extração dos dados com relação às características metodológicas dos estudos, intervençôes e resultados; discordâncias também foram resolvidas por consenso. Os principais resultados extraídos estavam relacionados à prevenção e ao manejo de náuseas e vômitos, neuropatia, toxicidade geral, caquexia, nefropatia e depressão. 
O risco de viés dos estudos incluídos foi avaliado de forma independente pelos dois revisores. Os artigos obedeceram aos seguintes critérios: 1 - Identificação de um problema clínico; 2 - Formulação de uma questáo clínica relevante e específica; 3 - Busca das evidências científicas; 4 - Avaliação das evidências disponíveis. Para essas questóes, foram utilizadas as informaçōes da Cochrane Effective Practice and Organization of Care ${ }^{10}$.

\section{RESULTADOS}

Após a fase de identificação dos artigos nas bases de dados, foi possível selecionar 455 títulos. Por meio da metodologia PRISMA, realizou-se a seleçâo em cada etapa (Figura 1). Da etapa de identificação à de seleção, foram excluídos 429 títulos, entre artigos repetidos, resumos e eventos científicos. Partindo dos 26 artigos remanescentes dos critérios de elegibilidade, foram incluídos 15 artigos após análise detalhada sobre o conteúdo de cada título encontrado na fase de elegibilidade. Esse número de artigos incluídos na revisão sistemática perfez 3\% do total de artigos encontrados nas bases de dados.

A coleta de dados foi distribuída em planilha estruturada, de acordo com os critérios da qualidade da evidência de Oxford $^{11}$ (Tabela 1), com os devidos parâmetros: autor, desenho do estudo, número de pacientes, neoplasia, qualidade da evidência.

De acordo com os estudos selecionados para revisão, buscaram-se algumas estratégias de prevenção ou manejo de RAM. Cada doença identificada com seu respectivo protocolo foi correlacionada com o tipo de RAM e o desfecho da intervenção (Tabela 2).

O presente estudo de revisão buscou verificar evidências científicas sobre manejo das principais reaçóes adversas causadas por tratamento com platinas em câncer gástrico e de esôfago. Foi possível identificar que os principais achados estavam relacionados a neuropatia, depressão, caquexia, reaçóes do trato gastrointestinal, mucosite, mielossupressão, diminuição dos niveis de magnésio e náusea e vômitos.

\section{NEUROPATIA}

Foram identificados três estudos que abordavam a neuropatia, dois se referiam a tratamentos farmacológicos e um a medidas não farmacológicas. Os estudos farmacológicos demonstram que o uso da venlafaxina ou da duloxetina favorece uma melhor resposta em todos os graus de neuropatia. Apesar de a N-acetilcisteína (NAC) ter obtido resultados favoráveis contra a neuropatia, o seu uso carece de mais estudos para comprovar sua eficácia. $\mathrm{O}$

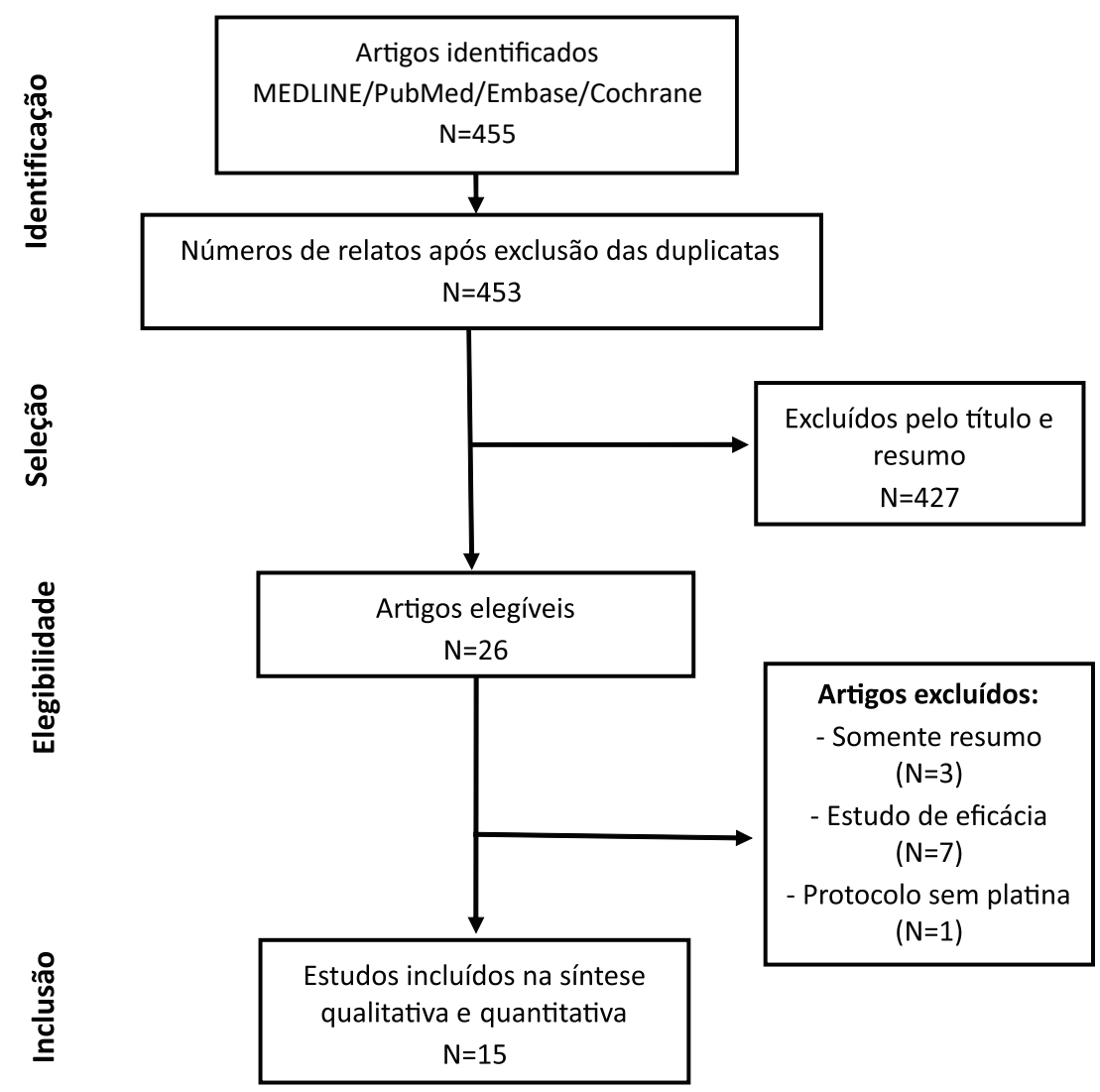

Figura 1. Fluxograma do processo de seleção de artigos seguindo a diretriz PRISMA 
Tabela 1. Descrição dos artigos selecionados

\begin{tabular}{|c|c|c|c|c|}
\hline Autor & $\begin{array}{l}\text { Desenho do } \\
\text { estudo }\end{array}$ & População amostral (N) & Neoplasia & $\begin{array}{c}\text { Qualidade } \\
\text { da } \\
\text { evidência }\end{array}$ \\
\hline Bondad et al. ${ }^{12}$ & $\begin{array}{l}\text { Ensaio clínico duplo- } \\
\text {-cego, randomizado }\end{array}$ & 32 & Câncer gástrico & A \\
\hline Eltweri et al. ${ }^{13}$ & $\begin{array}{l}\text { Ensaio clínico aberto } \\
\text { e randomizado }\end{array}$ & 57 & $\begin{array}{l}\text { Cânceres gástrico e } \\
\text { gastroesofágico }\end{array}$ & B \\
\hline $\begin{array}{l}\text { Farshchian et } \\
\text { al. }^{14}\end{array}$ & $\begin{array}{l}\text { Ensaio clínico duplo- } \\
\text {-cego }\end{array}$ & $\begin{array}{l}156 \\
(\text { Câncer de esôfago = 3) }\end{array}$ & $\begin{array}{l}\text { Diversos tipos de } \\
\text { câncer** }^{*}\end{array}$ & A \\
\hline Hamai et al. ${ }^{15}$ & $\begin{array}{l}\text { Ensaio clínico aberto } \\
\text { e randomizado }\end{array}$ & 18 & Câncer de esôfago & B \\
\hline Herrstedt et al. ${ }^{16}$ & Ensaio clínico aberto & 43 & Gastroesofágico & B \\
\hline Kaidarova et al. ${ }^{17}$ & $\begin{array}{l}\text { Ensaio clínico aberto } \\
\text { e não randomizado }\end{array}$ & $\begin{array}{l}127 \\
\text { (Câncer gástrico = 49) }\end{array}$ & $\begin{array}{l}\text { Câncer gástrico } \\
\text { e câncer de pulmão }\end{array}$ & B \\
\hline Karthaus et al. ${ }^{18}$ & $\begin{array}{l}\text { Ensaio clínico duplo- } \\
\text {-cego, randomizado }\end{array}$ & $\begin{array}{l}738 \\
\text { (Câncer gástrico = 49) }\end{array}$ & $\begin{array}{l}\text { Diversos tipos de } \\
\text { câncer** }\end{array}$ & A \\
\hline Khemissa et al. ${ }^{19}$ & $\begin{array}{l}\text { Ensaio clínico duplo- } \\
\text {-cego, randomizado }\end{array}$ & $\begin{array}{l}201 \\
\text { (Câncer gástrico = 17; } \\
\text { câncer de esôfago = 13) }\end{array}$ & $\begin{array}{l}\text { Câncer } \\
\text { gastrointestinal* }\end{array}$ & A \\
\hline Konishi et al. ${ }^{2}$ & $\begin{array}{l}\text { Ensaio clínico aberto } \\
\text { e não randomizado }\end{array}$ & 55 & Câncer de esôfago & B \\
\hline Kouchaki et al. ${ }^{20}$ & $\begin{array}{l}\text { Ensaio clínico duplo- } \\
\text {-cego, randomizado }\end{array}$ & $\begin{array}{l}90 \\
\text { (Câncer gástrico }=47 ; \\
\text { câncer de esôfago }=14 \text { ) }\end{array}$ & $\begin{array}{l}\text { Câncer } \\
\text { gastrointestinal* }\end{array}$ & A \\
\hline $\begin{array}{l}\text { Schönsteiner et } \\
\text { al. }^{21}\end{array}$ & $\begin{array}{l}\text { Ensaio clínico aberto } \\
\text { e randomizado }\end{array}$ & $\begin{array}{l}94 \\
\text { (Câncer gastroesofágico = 12) }\end{array}$ & $\begin{array}{l}\text { Diversos tipos de } \\
\text { câncer** }\end{array}$ & B \\
\hline $\begin{array}{l}\text { Schwartzberg et } \\
\text { al. }{ }^{22}\end{array}$ & $\begin{array}{l}\text { Ensaio clínico duplo- } \\
\text {-cego, randomizado }\end{array}$ & $\begin{array}{l}404 \\
\text { (Câncer gástrico = 24) }\end{array}$ & $\begin{array}{l}\text { Diversos tipos de } \\
\text { câncer** }\end{array}$ & A \\
\hline Song et al. ${ }^{23}$ & $\begin{array}{l}\text { Ensaio clínico duplo- } \\
\text {-cego, randomizado }\end{array}$ & $\begin{array}{l}83 \\
\text { (Câncer gástrico = 13; } \\
\text { câncer de esôfago = 13) }\end{array}$ & $\begin{array}{l}\text { Diversos tipos de } \\
\text { câncer** }\end{array}$ & A \\
\hline Tanaka et al. ${ }^{24}$ & $\begin{array}{l}\text { Ensaio clínico aberto } \\
\text { e não randomizado }\end{array}$ & 14 & Câncer de esôfago & B \\
\hline Yeganeh et al. ${ }^{25}$ & $\begin{array}{l}\text { Ensaio clínico aberto } \\
\text { e randomizado }\end{array}$ & $\begin{array}{l}62 \\
\text { (Câncer gástrico = 24; } \\
\text { câncer do esôfago }=5 \text { ) }\end{array}$ & $\begin{array}{l}\text { Diversos tipos de } \\
\text { câncer** }\end{array}$ & B \\
\hline
\end{tabular}

(*) Câncer gastrointestinal, incluindo câncer gástrico e/ou de esôfago.

$(* *)$ Vários tipos, incluindo câncer gástrico e/ou de esôfago.

estudo não farmacológico utilizando o método de vibração de corpo inteiro náo conseguiu demonstrar diferença estatística em relação ao grupo padrão ${ }^{12-14}$.

\section{DEPRESSÃO}

Um estudo sobre exercícios físicos e metabolismo da quinurenina (Kyn) mostrou que o exercício pode melhorar a depressão em pacientes com câncer. Os escores de depressão e ansiedade foram obtidos usando o questionário da Escala Hospitalar de Ansiedade e Depressão (HADS), além da coleta de amostras biológicas para verificação de concentraçôes plasmáticas de triptofano (Trp), Kyn, entre outros parâmetros. Ao longo do período de intervenção, ambos os grupos tiveram reduçóes significativas nos escores de ansiedade HADS ${ }^{15}$.

\section{CAQUEXIA, REAÇÕES DO TRATO GASTROINTESTINAL, MUCOSITE E MIELOSSUPRESSÃO}

Em um estudo que comparou pacientes elegíveis que receberam aleatoriamente megestrol mais placebo com megestrol mais celecoxibe e os resultados em relaçáo ao início do estudo, ambos os grupos revelaram uma melhora 
Tabela 2. Estratégias de prevenção e manejo de reações adversas à quimioterapia

\begin{tabular}{|c|c|c|c|c|}
\hline Autor & $\begin{array}{l}\text { Tipo de câncer/ } \\
\text { Protocolo }\end{array}$ & $\begin{array}{c}\text { Tipo de reação } \\
\text { adversa }\end{array}$ & Prevenção/Manejo & Desfecho \\
\hline $\begin{array}{l}\text { Bondad et } \\
\text { al. }{ }^{12}\end{array}$ & $\begin{array}{l}\text { Câncer gástrico } \\
\text { XELOX }\end{array}$ & NP & $\begin{array}{l}\text { Administração de } \\
\text { dois comprimidos } \\
\text { efervescentes de NAC } \\
1.200 \mathrm{mg}, 1 \mathrm{~h} \text { antes } \\
\text { da administração de } \\
\text { oxaliplatina por } 8 \\
\text { ciclos }\end{array}$ & $\begin{array}{l}\text { Grupo tratado com NAC } \\
\text { apresentou NP em } 68,8 \% \\
\text { vs. } 100 \% \text { de ocorrência no } \\
\text { grupo controle }\end{array}$ \\
\hline $\begin{array}{l}\text { Schönsteiner } \\
\text { et al. }{ }^{21}\end{array}$ & $\begin{array}{l}\text { Câncer gástrico e } \\
\text { câncer de esôfago } \\
\text { Protocolos à base de } \\
\text { cisplatina }\end{array}$ & NP & $\begin{array}{l}\text { Alongamentos, } \\
\text { mobilização passiva, } \\
\text { massagem e WBC. } \\
\text { Divididos em } 3 \text { etapas } \\
\text { e de acordo com } \\
\text { a tolerabilidade. } \\
\text { Intervalo de } 15 \text { dias } \\
\text { em um total de } 15 \\
\text { intervenções }\end{array}$ & $\begin{array}{l}\text { De acordo com o teste } \\
\text { da elevação da cadeira, } \\
\text { o grupo controle obteve } \\
\text { uma melhora de } 56 \% \text { vs. } \\
68 \% \text { do grupo teste (sem } \\
\text { diferença estatística). Na } \\
\text { NP, nos pés, houve uma } \\
\text { melhor resposta no grupo } \\
\text { teste na diminuição dos } \\
\text { sintomas de } 98 \% \text { para } 71 \% \\
\text { vs. } 97 \% \text { para } 81 \% \text {, grupo } \\
\text { controle (sem significância } \\
\text { estatística) }\end{array}$ \\
\hline $\begin{array}{l}\text { Farshchian } \\
\text { et. al. }{ }^{14}\end{array}$ & $\begin{array}{l}\text { Câncer de esôfago } \\
\text { FOLFOX } \\
\text { Carboplatina } \\
\text { TPF }\end{array}$ & NP & $\begin{array}{l}\text { Administração diária } \\
\text { de um comprimido de } \\
\text { venlafaxina } 37,5 \mathrm{mg} \\
\text { ou duloxetina } 30 \mathrm{mg}\end{array}$ & $\begin{array}{l}\text { Reações em seus maiores } \\
\text { graus } \\
\text { - NPC } \\
\text { - NP } \\
\text { - NPM } \\
\text { - NPS } \\
\text { - DNP } \\
\text { O grupo tratado com } \\
\text { venlafaxina vs. duloxetina } \\
\text { vs. placebo apresentou } \\
\text { respectivamente NPC } \\
21,6 \% \text { (grau 2) vs. 11,8\% } \\
\text { (grau 2) vs. 31,4\% (grau 2) } \\
\text { NPM 11,8\% (grau 3) vs. 0\% } \\
\text { (grau 3) vs. 17,6\% (grau 3) } \\
\text { NPS 5,9\% (grau 3) vs. 0\% } \\
\text { (grau 3) vs. 37,3\% (grau 3) } \\
\text { DNP 5,9\% (grau 2) vs. 0\% } \\
\text { (grau 3) vs. 11,8\% } \\
\text { (grau 3) }\end{array}$ \\
\hline $\begin{array}{l}\text { Herrstedt et } \\
\text { al. }{ }^{16}\end{array}$ & $\begin{array}{l}\text { Câncer } \\
\text { gastroesofágico }\end{array}$ & Depressão & $\begin{array}{l}\text { Exercícios físicos } \\
\text { aeróbico e de } \\
\text { resistência durante } \\
30 \text { a } 45 \text { min por } 12 \\
\text { semanas }\end{array}$ & $\begin{array}{l}\text { Grupo tratado com } \\
\text { atividade física apresentou } \\
\text { uma escala HADS de }-1,33 \\
{[2,36 ; 0,31], p=0,01 \text {. Com }} \\
\text { a média de concentração } \\
\text { de } 3 \text {-hidroxiquinurenina, } \\
\text { aumentou em } 48 \% \\
\text { ( } p=0,001 \text { ), enquanto } \\
\text { no grupo de exercício o } \\
\text { acúmulo dessa substância } \\
\text { foi atenuado }\end{array}$ \\
\hline
\end{tabular}


Tabela 2. continuação

\begin{tabular}{|c|c|c|c|c|}
\hline Autor & $\begin{array}{l}\text { Tipo de câncer/ } \\
\text { Protocolo }\end{array}$ & $\begin{array}{c}\text { Tipo de reação } \\
\text { adversa }\end{array}$ & Prevenção/Manejo & Desfecho \\
\hline $\begin{array}{l}\text { Kaidarova et } \\
\text { al. }{ }^{17}\end{array}$ & $\begin{array}{l}\text { Câncer gástrico } \\
\text { XELOX } \\
\text { Carboplatina + } \\
\text { paclitaxel }\end{array}$ & $\begin{array}{l}\text { Mielossupressão, } \\
\text { hepatotoxicidade, } \\
\text { nefrotoxicidade, } \\
\text { hipoalbuminemia, } \\
\text { astenia e } \\
\text { depressão }\end{array}$ & $\begin{array}{l}\text { Suplemento } \\
\text { alimentar composto } \\
\text { por aminoácidos, } \\
\text { vitaminas, minerais e } \\
\text { antioxidantes } 25 \mathrm{~mL} \text {, } \\
2 \text { vezes ao dia por } 20 \\
\text { dias }\end{array}$ & $\begin{array}{l}\text { Melhora significativa da } \\
\text { qualidade de vida (média, } \\
2,07 ; \text { IC } 95 \%, 1,00-4,29 \text { ). } \\
\text { Albumina mais elevada no } \\
\text { grupo teste (média, 38, } 1 \text {; } \\
\text { IC } 95 \%, 37,1-39,1 \mathrm{~g} / \mathrm{L} ; \mathrm{vs.} \\
\text { média do grupo controle, } \\
35,5 ; \text { IC de } 95 \%, 33,9- \\
37,0 ; p=0,03 \text { ) }\end{array}$ \\
\hline $\begin{array}{l}\text { Tanaka et } \\
\text { al. }^{24}\end{array}$ & $\begin{array}{l}\text { Câncer de esôfago } \\
\text { DCF }\end{array}$ & MO & $\begin{array}{l}\text { Glutamina } 8.832 \\
\mathrm{mg} / \text { dia e DE } 160 \mathrm{~g} . \\
\text { Uma semana antes e } \\
\text { continuando após o } \\
\text { início da quimioterapia }\end{array}$ & $\begin{array}{l}\text { A incidência de grau } \geq 2 \\
\text { MO no grupo que concluiu } \\
\text { a } D E \text { foi menor, } 15,4 \% \text { vs. } \\
66,7 \%(p=0,046) \text { dos que } \\
\text { não concluíram a DE }\end{array}$ \\
\hline $\begin{array}{l}\text { Khemissa et } \\
\text { al. }{ }^{19}\end{array}$ & $\begin{array}{l}\text { Câncer gástrico e } \\
\text { câncer de esôfago } \\
\text { Protocolos à base de } \\
\text { cisplatina ou } \\
\text { oxaliplatina }\end{array}$ & $\begin{array}{l}\text { Toxicidade geral } \\
\text { (gastrointestinal, } \\
\text { cutânea e } \\
\text { neurológica) }\end{array}$ & $\begin{array}{l}150 \mathrm{~g} \text { de suplemento } \\
\text { alimentar contendo } \\
\text { aproximadamente } \\
\text { glutamina } 13,5 \mathrm{~g} \\
\text { e TGF- } \beta 220 \mathrm{mg} \\
\text { (Clinutren } ® \text { ). Duas } \\
\text { tomadas diárias } \\
\text { (75 g cada) e } \\
\text { administrado durante } \\
5 \text { dias após o início da } \\
\text { quimioterapia }\end{array}$ & $\begin{array}{l}\text { As toxicidades não } \\
\text { hematológicas e } \\
\text { hematológicas de grau } 3-4 \\
\text { não foram estatisticamente } \\
\text { diferentes: } 22,6 \% \text { vs. } \\
19,2 \% \text { para as toxicidades } \\
\text { não hematológicas e } \\
17,7 \% \text { vs. } 15,2 \% \text { para as } \\
\text { toxicidades hematológicas, } \\
\text { nos grupos de controle vs. } \\
\text { grupo teste }\end{array}$ \\
\hline $\begin{array}{l}\text { Eltweri et } \\
\text { al. }{ }^{13}\end{array}$ & $\begin{array}{l}\text { Câncer } \\
\text { gastroesofágico } \\
\text { EOX }\end{array}$ & Toxicidade geral & $\begin{array}{l}\text { Ômega-3, } 2 \mathrm{~mL} / \mathrm{Kg} \text {, } \\
\text { em } 4 \mathrm{~h} \text { de infusão, } \\
\text { semanalmente }\end{array}$ & $\begin{array}{l}\text { Náusea/vômito no grupo } \\
\text { teste vs. grupo controle } \\
(0 \% \text { vs. } 19 \%, 260, p=0,04) \\
\text { Eventos tromboembólicos, } \\
\text { o grupo teste vs. controle } \\
(19 \% \text { vs. } 0 \%, p=0,04) \\
\text { Neutropenia de grau } 3 \text { ou } \\
4 \text { e grupo teste vs. controle } \\
(85 \% \text { vs. } 40 \%, p=0,002 \text { e } \\
60 \% \text { vs. } 16 \%, p<0,001)\end{array}$ \\
\hline $\begin{array}{l}\text { Kouchaki et } \\
\text { al. }{ }^{20}\end{array}$ & $\begin{array}{l}\text { Câncer gástrico e } \\
\text { câncer de esôfago } \\
\text { FOLFOX } \\
\text { XELOX } \\
\text { DCF } \\
\text { DOF }\end{array}$ & Caquexia & $\begin{array}{l}\text { Megestrol } 320 \text { mg/dia } \\
\text { + celcoxibe } 200 \text { mg/ } \\
\text { dia vs. megestrol } 320 \\
\text { mg/dia. Uso contínuo } \\
\text { na fase de estudo }\end{array}$ & $\begin{array}{l}\text { Após dois meses, os } \\
\text { pacientes no braço } 1 \text { (MA } \\
+ \text { placebo) e no braço } 2 \\
\text { (MA + celecoxib) obtiveram } \\
\text { ganho de peso de } 4,0 \\
\pm 3,4 \text { e } 2,2 \pm 3,6 \mathrm{~kg}, \\
\text { respectivamente }(\mathrm{p}=0,163)\end{array}$ \\
\hline $\begin{array}{l}\text { Konishi et } \\
\text { al. }^{2}\end{array}$ & $\begin{array}{l}\text { Câncer de esôfago } \\
\text { DCF } \\
\text { FP }\end{array}$ & $\begin{array}{l}\text { Nefrotoxicidade. } \\
\text { Diminuição da } \\
\text { taxa de filtração } \\
\text { glomerular }\end{array}$ & $\begin{array}{l}\text { Suplementação de } \mathrm{Mg} \\
\text { intravenoso, } 8 \mathrm{mg} \text { no } \\
\text { D1 da quimioterapia }\end{array}$ & $\begin{array}{l}\text { Aumento das concentrações } \\
\text { de creatinina pós- } \\
\text {-quimioterapia sem } \\
\text { suplementação de } \mathrm{Mg} \\
\text { ( } p=0,01 \text { ), com aumento } \\
\text { de graus } 1 \text { e } 2 \text { de } 22,2 \% \\
\text { e } 5,6 \% \text {, respectivamente. } \\
\text { Após suplementação de } \mathrm{Mg} \\
\text { (alteração na creatinina, } \\
\text { p=0,21), com aumento de } \\
\text { graus } 1 \text { e } 2 \text { de } 8,1 \% \text { e } 0 \% \text {, } \\
\text { respectivamente }\end{array}$ \\
\hline
\end{tabular}


Tabela 2. continuação

\begin{tabular}{|c|c|c|c|c|}
\hline $\begin{array}{l}\text { Yeganeh et } \\
\text { al. }{ }^{25}\end{array}$ & $\begin{array}{l}\text { Câncer gástrico e } \\
\text { câncer de esôfago } \\
\text { Protocolos com } \\
\text { cisplatina }\end{array}$ & Hipomagnesemia & $\begin{array}{l}\text { Suplementação de } \mathrm{Mg} \\
\text { oral, } 500 \mathrm{mg} \text { para cada } \\
50 \mathrm{mg} / \mathrm{m}^{2} \text { de cisplatina, } \\
\text { dividido em } 2 \text { ou } 3 \\
\text { tomadas diárias após } \\
\text { a conclusão de cada } \\
\text { ciclo, continuando até } \\
\text { o próximo ciclo, e } 2 \text { a } 3 \\
\text { semanas após o último } \\
\text { ciclo de quimioterapia }\end{array}$ & $\begin{array}{l}\text { Após acompanhamento, } \\
\text { a prevalência de } \\
\text { hipomagnesemia no grupo } \\
\text { de intervenção foi de } 10,7 \% \\
\text { vs. } 23,1 \% \text { no grupo controle }\end{array}$ \\
\hline $\begin{array}{l}\text { Karthaus et } \\
\text { al. }{ }^{18}\end{array}$ & $\begin{array}{l}\text { Câncer gástrico } \\
\text { Protocolos à base de } \\
\text { cisplatina }\end{array}$ & Náusea e vômito & $\begin{array}{l}\text { Palonasetrona } \\
\text { oral, 0,5 mg vs. } \\
\text { palonasetrona IV, 0,25 } \\
\text { mg. Dexametasona } \\
20 \text { mg no D1, seguido } \\
\text { de dexametasona } 8 \\
\text { mg do D2 ao D4 para } \\
\text { ambos os grupos. Os } \\
\text { medicamentos eram } \\
\text { administrados na } \\
\text { antes e 24h após a } \\
\text { quimioterapia }\end{array}$ & $\begin{array}{l}\text { A taxa de resposta completa } \\
\text { na fase aguda foi de } 89,4 \% \\
\text { para o oral vs. } 86,2 \% \text { para } \\
\text { o IV. O intervalo de não } \\
\text { inferioridade foi de } 3,21 \% \\
\text { (IC de } 99 \% \text { ) }\end{array}$ \\
\hline Song et al. ${ }^{23}$ & $\begin{array}{l}\text { Câncer gástrico e } \\
\text { câncer de esôfago } \\
\text { Protocolos à base de } \\
\text { cisplatina }\end{array}$ & Náusea e vômito & $\begin{array}{l}\text { Talidomida } 100 \mathrm{mg} \text {, } \\
\text { dexametasona } 4,5 \mathrm{mg} \\
\text { e metoclopramida } 10 \\
\text { mg, todos VO do D1 } \\
\text { ao D5 }\end{array}$ & $\begin{array}{l}\text { Taxa de resposta aguda } \\
\text { da talidomida em relação } \\
\text { ao grupo controle }(93 \% \\
\text { vs. } 91 \%, p=0,767) \text {. A taxa } \\
\text { de resposta completa foi } \\
\text { mais alta para o grupo } \\
\text { de talidomida durante a } \\
\text { fase geral (75\% vs. } 51 \% \text {, } \\
p=0,024)\end{array}$ \\
\hline Hamai et al. ${ }^{15}$ & $\begin{array}{l}\text { Câncer de esôfago } \\
\text { Protocolos à base de } \\
\text { cisplatina }\end{array}$ & Náusea e vômito & $\begin{array}{l}\text { RKT, } 7,5 \mathrm{~g} \text { /dia por } \\
14 \text { dias, começando } \\
\text { no D1 da cisplatina. } \\
\text { Associados aos } \\
\text { protocolos padrões de } \\
\text { inibidores de } 5-\mathrm{HT} 3 \text {, } \\
\text { inibidores de NK1 e } \\
\text { corticosteroides }\end{array}$ & $\begin{array}{l}\text { A taxa média em } \\
\text { na ingestão de alimentos } \\
\text { diminuiu entre os dias } 4 \text { e } \\
6 \text { e foi consideravelmente } \\
\text { menor no curso com RKT } \\
\text { do que sem o fitoterápico } \\
(2 \% \text { vs. } 30 \%, p=0,01, \\
\text { respectivamente) }\end{array}$ \\
\hline $\begin{array}{l}\text { Schwartzberg } \\
\text { et al. }{ }^{22}\end{array}$ & $\begin{array}{l}\text { Câncer gástrico } \\
\text { Protocolos com } \\
\text { cisplatina e outros } \\
\text { agentes alquilantes }\end{array}$ & Náusea e vômito & $\begin{array}{l}\text { NEPA: netupitanto } 300 \\
\text { mg e palonasetrona } \\
0,25 \text { mg (NEPA oral), } \\
\text { via oral, } 60 \text { minutos } \\
\text { antes da quimioterapia } \\
\text { vs. fosnetupitanto } 235 \\
\text { mg e palonasetrona } \\
0,25 \text { mg (NEPA IV), via } \\
\text { intravenosa, } 30 \text { minutos } \\
\text { antes da quimioterapia. } \\
\text { Dexametasona } 12 \text { mg } \\
\text { no D1, seguido de } \\
\text { dexametasona } 8 \text { mg do } \\
\text { D2 ao D4 antes da QT } \\
\text { para ambos os grupos }\end{array}$ & $\begin{array}{l}\text { Tanto no NEPA IV quanto } \\
\text { VO, a incidência de eventos } \\
\text { adversos relacionados ao } \\
\text { tratamento foi semelhante } \\
\text { em ambos os grupos ( } 12,8 \% \\
\text { NEPA IV e } 11,4 \% \text { NEPA oral } \\
\text { durante todo o estudo) }\end{array}$ \\
\hline
\end{tabular}

Legendas: NP = Neuropatia; IV = Intravenoso; VO = Via oral; XELOX = Capecitabina/oxaliplatina; FOLFOX = Folinato de cálcio/fluorouracil/oxaliplatina; TPF = Paclitaxel/cisplatina/fluorouracil; EOX = Epirubicina/oxaliplatina/capecitabina; ECX = Epirubicina/cisplatina/capecitabina; CROSS = Paclitaxel/carboplatina/ radioterapia; DCF $=$ Docetaxel/cisplatina/fluorouracil; DOF $=$ Docetaxel/oxaliplatina/fluorouracil; FP $=$ Fluorouracil/cisplatina; $\mathrm{DE}=\mathrm{Dieta}$ elementar; $\mathrm{QT}=$ Quimioterapia; RKT $=$ Fitoterápico Rikkunshito; vs. $=$ Versus; NPC = NP craniana; NPM = NP motora; NPS = NP sensorial; $\mathrm{DNP}=\mathrm{Dor}$ neuropática; $\mathrm{D}=$ dia; $\mathrm{MO}$ = Mucosite oral; IC = Intervalo de confiança; WBC = Vibração de corpo inteiro; HADS = Hospitalar de Ansiedade e Depressão; TGF- $\beta 2$ = Fator de transformação do crescimento beta 2; $\mathrm{Mg}$ = Magnésio; NEPA = Netupitanto/palonosetrona; NAC = N-acetilcisteína; $\mathrm{MA}=$ Acetato de megestrol. 
significativa, porém a avaliação final mostrou que a adição de celecoxibe ao megestrol náo aumentou os efeitos anticaquexia do megestrol ${ }^{20}$.

Dois trabalhos clínicos fizeram uso de compostos nutricionais, tais como: oncoxina (ONCX), glutamina, fator de transformaçáo do crescimento beta 2 (TGF- $\beta 2$ ) e ácidos graxos poli-insaturados de ômega-3 (PUFA ômega-3). O ONCX foi utilizado com objetivo de diminuir os efeitos colaterais, aumentando a QV. Embora os resultados obtidos pareçam promissores, mais estudos de suplementos nutricionais multicomponentes são necessários para explorar oportunidades de melhorar a QV dos pacientes ${ }^{26}$.

Um ensaio clínico de fase II sobre suplementação alimentar investigou os efeitos clínicos, radiológicos e de citocinas de infusão intravenosa de PUFA ômega-3. Os achados mais significativos foram uma frequência reduzida de toxicidade gastrointestinal e eventos tromboembólicos. Nenhuma vantagem de sobrevivência foi demonstrada para aqueles tratados com epirubicina/oxaliplatina/ capecitabina (EOX) mais óleo de peixe (PUFA ômega-3). Os autores concluíram que esse benefício na taxa de resposta e redução de eventos adversos gastrointestinais relacionados à quimioterapia em $\mathrm{EOX}$, com ou sem óleo de peixe, deve ser avaliado pelo menos em um estudo de fase II randomizado ${ }^{19}$.

Por fim, um estudo envolvendo pacientes com carcinoma espinocelular ou adenocarcinoma esofágico estágios II/III seguiu o protocolo docetaxel/cisplatina/ fluorouracil (DCF). Os autores concluíram que uma dieta elementar (DE) pode ser um dos tratamentos de teste para reduzir a incidência de mucosite oral $(\mathrm{MO})$ e deve ser avaliada em outro estudo randomizado ${ }^{17}$.

\section{SUPLEMENTAÇÃO COM MAGNÉSIO}

Dois estudos sobre suplementação de magnésio $(\mathrm{Mg})$ foram identificados, sendo um por via intravenosa e outro por via oral. No trabalho de suplementação por via intravenosa, foi administrado $\mathrm{Mg}$ em pacientes com carcinoma espinocelular de esôfago tratados com um regime de alta dose contendo cisplatina, e os efeitos protetores da suplementação de $\mathrm{Mg}$ contra a nefrotoxicidade induzida pela cisplatina foram examinados prospectivamente em relaçáo aos níveis de paratormônio (PTH) e proteína relacionada ao paratormônio (PTH-rP). Nenhum paciente apresentou desnutrição ou desidratação durante o tratamento ${ }^{2}$.

No estudo relacionado à suplementação de $\mathrm{Mg}$ por via oral, houve efeito significativo em relaçáo ao grupo controle da suplementaçáo de acordo com a dose de cisplatina, contribuindo na redução do declínio nos níveis $\mathrm{Mg}$ sérico após os seis ciclos de quimioterapia à base de cisplatina ${ }^{21}$.

\section{NÁUSEAS EVÔMITOS}

$\mathrm{Na}$ prevenção de náuseas e vômitos, foi possível identificar quatro estudos relacionados a pacientes com cânceres gástrico e esofágico, sendo dois deles relacionados ao uso da associação de inibidores de serotonina 5-HT3 e inibidores de neurocininas $N K 1^{4,23}$, um relacionado ao uso da talidomida e um produto natural fitoterápico conhecido como Rikkunshito (RKT) ${ }^{22-24}$. De acordo com os estudos, os resultados sugerem um perfil satisfatório na resposta contra náusea e vômito, conforme demonstrado na Tabela 2.

\section{DISCUSSÃO}

As principais RAM observadas nos estudos foram a ocorrência de neuropatia, depressão, náuseas e vômitos, hipomagnesemia, toxicidades gastrointestinais, caquexia e mucosite. As abordagens apontadas nesses estudos envolveram condutas farmacológicas e não farmacológicas.

Derivados da platina (oxaliplatina, cisplatina e carboplatina) são agentes alquilantes que inibem a síntese e a replicação do DNA por meio de ligaçóes cruzadas estabelecidas pelo complexo de platina ${ }^{4}$. As RAM experimentadas por pacientes tratados com os medicamentos antineoplásicos são semelhantes, embora a toxicidade limitadora de dose específica (DLT) seja diferente para cada fármaco. Para a cisplatina, a DLT é a nefrotoxicidade; para carboplatina, mielossupressão; e, para oxaliplatina, neurotoxicidade ${ }^{26}$.

Mecanismos distintos de neuropatia têm sido propostos para as diferentes classes de antineoplásicos. Os derivados de platinas podem reduzir o transporte axonal e, consequentemente, induzir apoptose de neurônios sensoriais. Além disso, estudos experimentais mostram acúmulo de compostos de platina nos corpos celulares dos gânglios da raiz dorsal, o que resulta na diminuição do metabolismo celular e no transporte axonal. Parecem ocorrer também lesóes mitocondriais com aumento do estresse oxidativo, o que induziria a ocorrência de neuropatia crônica ${ }^{22}$.

Em três estudos com abordagem de tratamentos diferentes para prevenção de neuropatias, foram avaliados 47 pacientes, em que foi possível observar respostas diversas de acordo com a conduta terapêutica adotada. O estudo com os medicamentos venlafaxina e duloxetina, entre os tratamentos observados, parece ser o com maior nível de evidência. Este ensaio clínico contou com um total de indivíduos igual a 156. Destes, foram identificados três pacientes com câncer esofágico. Os resultados gerais demonstraram que os efeitos na diminuição da neuropatia motora e da dor neuropática foram melhores nos grupos da duloxetina e da venlafaxina. 
Após quatro semanas, observou-se que 23,5\% dos pacientes, para cada um desses medicamentos, não apresentaram sintomas de neurotoxicidade e nenhum do grupo da duloxetina teve reação de neuropatia de grau $3^{14}$. O possível benefício do uso de venlafaxina foi sugerido em um pequeno estudo controlado por placebo com 48 pacientes com de neuropatia aguda por oxaliplatina. O grupo da venlafaxina apresentou alívio dos sintomas quando comparado ao grupo que recebeu placebo (31\% versus 5\%), respectivamente. Em outro estudo, com 231 pacientes, relacionando o uso da duloxetina comparado com o placebo, o risco relativo de redução de $30 \%$ da dor com duloxetina foi de 1,96 (IC95\%=1,15-3,35) e o de reduçâo de $50 \%$ foi de 2,43 (IC95\%=1,11-5,30), além de ter melhorado também a $\mathrm{QV}^{27}$.

Em estudo conduzido por Bondad et al. ${ }^{12}$, de um total de 32 pacientes, 16 eram do grupo controle e 16 faziam uso de NAC. Apesar dos resultados positivos, 32,25\% dos pacientes do grupo de NAC não tiveram neuropatia. Vale destacar que os resultados eletrofisiológicos sensoriais não mostraram diferença significativa entre os dois grupos, controle e teste. Estudos por um período prolongado de uso são recomendados para confirmar o potencial papel da NAC ${ }^{25}$.

Finalizando a série de trabalhos clínicos relacionados à neuropatia, em um estudo com 94 pacientes $(12 \mathrm{com}$ câncer gastroesofágico), avaliou-se a neurotoxicidade com um método de terapia não medicamentosa, o WBC. Os autores sugerem que esse programa, comparado com terapias medicamentosas, incluindo antidepressivos, anticonvulsivantes, agentes antioxidantes, medicamentos neuroprotetores ou plantas medicinais, pode ter resposta objetiva nas neuropatias ${ }^{13}$.

A depressão aparece como uma doença comórbida em aproximadamente $25 \%$ de todos os pacientes com câncer. As pessoas que recebem um diagnóstico de câncer passam por vários níveis de estresse e angústia emocional, o que pode levar ao seu desencadeamento ${ }^{28}$.

Em uma pesquisa com 43 pacientes com câncer gastrointestinal, os autores consideraram que, em 12 semanas de exercícios supervisionados antes da cirurgia eletiva, foi possível reduzir os sintomas de depressão em pacientes com diagnóstico de câncer GEJ operável ${ }^{20}$. Um estudo clínico randomizado com Yoga em pacientes com câncer de mama $(n=88)$ corroborou os efeitos benéficos dos exercícios em pacientes com câncer e apontou para uma explicação potencial dos efeitos benéficos do exercício na depressão. Tanto a ansiedade quanto a depressão podem afetar o sofrimento relacionado ao tratamento, fazendo com que os pacientes percebam o câncer como uma ameaça, aumentando a atenção aos sintomas somáticos e causando sintomas aversivos ${ }^{29}$.
Todos os medicamentos de antineoplásicos, incluindo aqueles à base de platina, exibem uma gama de efeitos colaterais graves em razão da sua baixa seletividade para o tecido canceroso comparado ao tecido normal pela alta necessidade de nutrientes das células cancerosas. Embora esses fármacos sejam absorvidos por células cancerígenas de crescimento rápido, também são absorvidos por outros tecidos normais que estão crescendo rapidamente ${ }^{27}$.

No caso da mucosite, possui uma relação com os compostos reativos de oxigênio (ROS), que são os primeiros condutores de danos à mucosa e representam um alvo potencial para a inibiçấo do desenvolvimento da mucosite ${ }^{30}$.

De forma geral, os estudos envolvendo a glutamina nas diversas associaçóes ainda continuam contraditórios, principalmente com relaçáo à mucosite. Foram identificados quatro estudos com suplementação dietética compreendendo um total de 150 pacientes. Destes, 44 pacientes fizeram uso de glutamina em dois estudos diferentes para a prevenção de $\mathrm{MO}$ e toxicidade geral. O primeiro trabalho, com 30 pacientes de câncer gástrico e câncer de esôfago, não apoiou a hipótese de que a adição de glutamina e TGF- $\beta 2$ poderia prevenir ou diminuir as toxicidades não hematológicas de graus 3 e 4 relacionadas à quimioterapia em pacientes com câncer gastrointestinal. Segundo o autor, até o dado momento, nenhum estudo clínico randomizado foi publicado sobre o efeito do TGF- $\beta 2$ em pacientes com câncer e o benefício dos suplementos orais de glutamina em pacientes com câncer ainda é controverso. Além disso, toxicidades hematológicas, interrupçóes de tratamento e marcadores inflamatórios não foram diferentes entre os dois grupos ${ }^{18}$. A administraçáo de compostos eliminadores de ROS, como a glutamina, forneceram evidências contraditórias de sua eficácia na prevenção da mucosite ${ }^{30}$. Alguns estudos com números baixos de indivíduos tornam difícil a avaliação da $\mathrm{MO}$, necessitando de outros estudos randomizados e com um número de participantes maior para uma melhor evidência ${ }^{17,31}$.

Uma suplementação dietética com ONCX foi utilizada por 49 pacientes com câncer gástrico e avaliada por um grupo de pesquisadores com objetivo de diminuir a toxicidade da terapia relacionada à má QV. De acordo com os achados, foi possível observar que todos os parâmetros relacionados à função hepática (ALT e AST), à hemoglobina, ao estado emocional, à perda de apetite, ao estado físico e ao cansaço foram menores no grupo do medicamento em comparaçáo ao grupo controle ${ }^{16}$. Vitaminas que regulam a via do metabolismo de um carbono (piridoxina, folato e cobalamina) desempenham um papel crucial no DNA estabilização e reparação de estruturas. Juntas, essas propriedades podem ser 
responsáveis pela eficácia do ONCX na redução dos sintomas de $\mathrm{OM}$ e melhorar a capacidade de ingerir alimentos sólidos e manter peso corporal. Além de controlar complicações infecciosas e diminuírem a toxicidade da terapia no que diz respeito à contagem de leucócitos e danos ao fígado ${ }^{32}$.

Em estudo com 57 pacientes com câncer gástrico e de esôfago, relacionado à suplementação, foram utilizados PUFA ômega-3. Os autores concluíram que esse benefício na taxa de resposta e redução de eventos adversos gastrointestinais relacionados à quimioterapia com o protocolo EOX, com ou sem óleo de peixe, deve ser avaliado pelo menos em um estudo de fase II randomizado $^{19}$. Outro trabalho do mesmo autor revela que os PUFA, o DHA sozinho ou em combinação $\left(\right.$ Omegaven $\left.{ }^{\circledR}\right)$, tiveram maiores efeitos anticâncer in vitro do que o ácido eicosapentaenoico (EPA) sozinho. Os PUFA ômega-3 são EPA e ácido docosaexaenoico (DHA), que têm efeitos anti-inflamatórios, e apenas uma pequena quantidade desses PUFA pode ser sintetizada no corpo humano. Aproximadamente em 2-10\% de $\alpha$-linolênico, o ácido é convertido em EPA e DHA ${ }^{33}$ Os PUFA são componentes importantes das membranas celulares em virtude da sua fluidez. Essas moléculas são substratos para a produção de eicosanoides inflamatórios e anti-inflamatórios, como exemplificado pelas prostaglandinas e leucotrienos. Em células de mamíferos, os ácidos graxos ômega-6 e ômega-3 competem pelo metabolismo pela mesma enzima, produzindo ácido araquidônico ou EPA e DHA, respectivamente. EPA e DHA podem substituir o ácido araquidônico nas membranas celulares e suprimirem a produção de mediadores pró-inflamatórios ${ }^{34}$.

Um dos problemas importantes relacionados ao câncer é o manejo da caquexia. A caquexia afeta os domínios físico, psicológico e social de vida concomitantemente. Como resultado, a $\mathrm{QV}$ dos pacientes com caquexia diminui drasticamente. A evidência atual sugere que a caquexia se desenvolve após uma prolongada resposta inflamatória. O produto é o catabolismo de proteínas musculares enquanto o corpo tenta fornecer elementos necessários para a síntese de proteínas de fase aguda ${ }^{35-37}$. Um estudo randomizado e duplo-cego com 90 pacientes com cânceres gastrointestinais (destes, 47 com câncer gástrico e 14 com câncer de esôfago) demonstrou em seus resultados, em relaçáo ao início do estudo, entre os grupos controle e teste, melhora significativa no grupo teste, o que o fez diferente de alguns estudos-piloto e ensaios clínicos que relataram resultados encorajadores usando celecoxibe ou anti-inflamatórios tradicionais na caquexia, porém concluiu-se que os resultados não conseguiram mostrar que a adição de celecoxibe ao megestrol aumentou os efeitos anticaquexia do megestrol ${ }^{20}$. Em uma revisão sistemática que identificou 3.368 portadores de câncer (23\% destes tinham câncer gastrointestinal), foi possível observar que houve benefício com relação ao ganho de peso e aumento de apetite nos pacientes tratados com acetato de megestrol isolado ${ }^{38}$.

Como as platinas causam nefrotoxicidade, não é totalmente compreendido, mas acredita-se que seja por conta de danos diretos e indiretos aos rins. O principal mecanismo é atribuído à necrose tubular aguda das células tubulares proximais. Outro efeito das platinas, principalmente a cisplatina, é a hipomagnesemia, que decorre da incapacidade do rim danificado de reabsorver $\mathrm{Mg}^{26}$. Dois estudos com 117 pacientes abordaram a suplementaçáo com o $\mathrm{Mg}$, com intuito de evitar a hipomagnesemia e a prevenção de reaçôes de nefrotoxicidade. Um deles realizou um teste, embora em pequena escala, que revelou que a suplementação intravenosa de $\mathrm{Mg}$ conferiu efeitos protetores contra a nefrotoxicidade induzida pela cisplatina em pacientes com carcinoma de células escamosas de esôfago; o outro, com uso de $\mathrm{Mg}$ oral, sugeriu mais investigaçôes para estabelecer uma diretriz útil para a suplementação de $\mathrm{Mg}$ em pacientes recebendo quimioterapia à base de cisplatina ${ }^{2,25}$. Estudos apontam que, em alguns pacientes, a cisplatina pode induzir um defeito na conservação tubular renal de $\mathrm{Mg}$, que pode resultar em síndromes clínicas graves de deficiência de $\mathrm{Mg}$, sendo a suplementação de eletrólitos uma forma de diminuir o risco de nefrotoxicidade após a quimioterapia com cisplatina ${ }^{39}$.

Um dos principais problemas relacionados aos protocolos contendo platinas são as reaçôes de náusea e vômitos. A cisplatina é considerada um medicamento de alto risco emético, com mais de $90 \%$ dos pacientes apresentando náuseas e vômitos. Em contraste, a carboplatina e a oxaliplatina são classificadas como medicamentos de risco moderado, com taxas de náusea e vômito entre 30-90\%. Se os pacientes também receberem radioterapia concomitante com seu medicamento de platina, isso pode aumentar a incidência de náusea e vômito induzida por quimioterapia (CINV). Embora o tratamento com medicamentos antieméticos seja amplamente bem-sucedido, 10-30\% dos pacientes ainda apresentam náuseas e vômitos. Quatro estudos foram selecionados. Um deles tratava da comparação entre o antiemético palonasetrona oral e o palonasetrona intravenoso, que tiveram perfis de segurança comparáveis sem levantar novas preocupaçóes ${ }^{18}$. Tanto a combinação de netupitanto e palonosetrona (NEPA) intravenoso quanto oral mostraram altas respostas na prevenção de CINV ${ }^{40,41}$. $\mathrm{O}$ estudo sobre a talidomida em doses mais baixas não aumentou a toxicidade da quimioterapia. No entanto, a dosagem ideal de talidomida no controle de CINV 
era incerta ${ }^{23,42}$. Por fim, com o fitoterápico RKT, não se observou nenhum evento adverso, além de melhorar a QV de pacientes com câncer de esôfago ${ }^{24}$. A combinação de um antagonista de receptores NK1, dexametasona e um antagonista de receptor 5-HT3 (5HT3RA) é atualmente considerada o antiemético padrão no tratamento de pacientes recebendo quimioterapia à base de cisplatina. A palonosetrona, um 5HT3RA de segunda geraçáo, demonstrou ser superior na prevenção de vômitos agudos e retardados em comparação aos 5HT3RA de primeira geração $^{43,44}$.

\section{CONCLUSÃO}

De acordo com os estudos aqui apresentados, foi possível identificar que náuseas e vômitos, neuropatia e hipomagnesemia tiveram um maior número de estudos relacionados ao manejo e à prevenção dessas reaçôes, nos quais apresentaram-se algumas sugestóes de condutas com maior evidência. São necessários mais estudos na prevenção e manejo adequado para reações como depressão, mucosite e caquexia, principalmente nos casos de câncer gástrico e câncer de esôfago.

\section{CONTRIBUIÇÕES}

Ney Moura Lemos Pereira contribuiu na concepção ou desenho do trabalho; na aquisição, análise, e/ou interpretaçáo dos dados da pesquisa; na redaçáo e/ ou revisão crítica com contribuição intelectual. Telma Maria Araújo Moura Lemos e Fernanda Nervo Raffin contribuíram na concepção ou desenho do trabalho; na aquisição, análise, e/ou interpretação dos dados da pesquisa. Rand Randall Martins e Roberto Fernandes da Costa contribuíram na redação e/ou revisão crítica. Todos os autores aprovaram a versão final a ser publicada.

\section{DECLARAÇÃO DE CONFLITO DE INTERESSES}

Nada a declarar.

\section{FONTES DE FINANCIAMENTO}

Coordenação de Aperfeiçoamento de Pessoal de Nível Superior (Capes) - Código de financiamento 001.

\section{AGRADECIMENTOS}

Aos médicos Marcos Dias Leão (Oncoclínica São Marcos, Natal-RN) e Andréa Juliana Pereira Gomes (Liga contra o Câncer - Natal-RN) pela revisão geral de terminologias, conteúdo clínico e terapêutico dos estudos incluídos na revisão. À farmacêutica Andréa
Pinto Fernandes (Liga contra o Câncer - Natal-RN) pela contribuição na interpretaçáo de reaçóes adversas e protocolos terapêuticos.

\section{REFERÊNCIAS}

1. Guo Q, Liu X, Lu L, et al. Comprehensive evaluation of clinical efficacy and safety of celecoxib combined with chemotherapy in management of gastric cancer. Medicine (Baltimore). 2017;96(51):e8857. doi: http:// doi.org/10.1097/MD.0000000000008857

2. Konishi $\mathrm{H}$, Fujiwara $\mathrm{H}$, Itoh $\mathrm{H}$, et al. Influence of magnesium and parathyroid hormone on cisplatin-induced nephrotoxicity in esophageal squamous cell carcinoma. Oncol Lett. 2018;15(1):658-64. doi: http://doi.org/10.3892/ol.2017.7345

3. Travis LB, Fossa SD, Sesso HD, et al. Chemotherapyinduced peripheral neurotoxicity and ototoxicity: new paradigms for translational genomics. J Natl Cancer Inst. 2014;106(5):dju044. doi: http://doi.org/10.1093/ jnci/dju044

4. Hartmann JT, Lipp HP. Toxicity of platinum compounds. Expert Opin Pharmacother. 2003;4(6):889-901. doi: http://doi.org/10.1517/14656566.4.6.889

5. Ruggiero A, Trombatore G, Triarico S, et al. Platinum compounds in children with cancer: toxicity and clinical management. Anticancer Drugs. 2013;24(10):1007-19. doi: http://doi.org/10.1097/CAD.0b013e3283650bda

6. Instituto Nacional de Câncer José Alencar Gomes da Silva. Estimativa 2020: incidência de câncer no Brasil. Rio de Janeiro: INCA; 2019.

7. National Cancer Institute (US). Division of Cancer Treatment and Diagnosis. Commom toxicity criteria [Computer Program]. Version 5.0. Bethesda (MD): $\mathrm{NIH} ; 2017$.

8. Moher D, Liberati A, Tetzlaff J, et al. Preferred reporting items for systematic reviews and meta-analyses: the PRISMA statement. PLoS Med. 2009;6(7):e1000097. doi: http://doi.org/10.1371/journal.pmed.1000097

9. Ministério da Saúde (BR), Secretaria de Ciência, Tecnologia e Insumos Estratégicos, Departamento de Ciência e Tecnologia. Diretrizes metodológicas: elaboração de revisão sistemática e metanálise de ensaios clínicos randomizados [Internet]. Brasília, DF: Ministério da Saúde; 2012 [acesso 2020 jul. 22]. Disponível em: http://bvsms.saude.gov.br/bvs/publicacoes/diretrizes_ metodologicas_elaboracao_sistematica.pdf

10. Cochrane Effective Practice and Organization of Care [Internet]. London: Cochrane; c2021. EPOC resources for review authors; 2017 [cited 2020 July 22]. Available from: https://epoc.cochrane.org/resources/epocresources-review-authors

11. Centre for Evidence-based Medicine [Internet]. Oxford (UK): University of Oxford; c2021. Oxford Centre for 
Evidence-Based Medicine: levels of evidence; Mar 2009 [cited 2020 July 22]. Available from: https://www.cebm. ox.ac.uk/resources/levels-of-evidence/oxford-centre-forevidence-based-medicine-levels-of-evidence-march-2009

12. Bondad N, Boostani R, Barri A, et al. Protective effect of $\mathrm{N}$-acetylcysteine on oxaliplatin-induced neurotoxicity in patients with colorectal and gastric cancers: a randomized, double blind, placebo-controlled, clinical trial. J Oncol Pharm Pract. 2020;26(7):1575-82. doi: http://doi.org/10.1177/1078155219900788

13. Eltweri AM, Thomas AL, Chung WY, et al. The effect of supplementary Omegaven ${ }^{\varpi}$ on the clinical outcome of patients with advanced esophagogastric adenocarcinoma receiving palliative epirubicin, oxaliplatin, and capecitabine chemotherapy: a phase II clinical trial. Anticancer Res. 2019;39(2):853-61. doi: http://doi. org/10.21873/anticanres.13185

14. Farshchian N, Alavi A, Heydarheydari S, et al. Comparative study of the effects of venlafaxine and duloxetine on chemotherapy-induced peripheral neuropathy. Cancer Chemother Pharmacol. 2018;82(5):787-93. doi: https:// doi.org/10.1007/s00280-018-3664-y

15. Hamai Y, Yoshiya T, Hihara J, et al. Traditional Japanese herbal medicine rikkunshito increases food intake and plasma acylated ghrelin levels in patients with esophageal cancer treated by cisplatin-based chemotherapy. J Thorac Dis. 2019;11(6):2470-78. doi: http://doi.org/10.21037/ jtd.2019.05.67

16. Herrstedt A, Baya ML, Simonsen C, et al. Exercisemediated improvement of depression in patients with gastro-esophageal junction cancer is linked to kynurenine metabolism. Acta Oncol. 2019;58(5):579-87. doi: https://doi.org/10.1080/0284186X.2018.1558371

17. Kaidarova DR, Kopp MV, PokrovskyVS. Multicomponent nutritional supplement Oncoxin and its influence on quality of life and therapy toxicity in patients receiving adjuvant chemotherapy. Oncol Lett. 2019;18(5):564452. doi: http://doi.org/10.3892/ol.2019.10868

18. Karthaus M, Tibor C, Lorusso V, et al. Efficacy and safety of oral palonosetron compared with IV palonosetron administered with dexamethasone for the prevention of chemotherapy-induced nausea and vomiting (CINV) in patients with solid tumors receiving cisplatin-based highly emetogenic chemotherapy (HEC). Support Care Cancer. 2015;23(10):2917-23. doi: http://doi. org/10.1007/s00520-015-2657-1

19. Khemissa F, Mineur L, Amsellem C, et al. A phase III study evaluating oral glutamine and transforming growth factor-beta 2 on chemotherapy-induced toxicity in patients with digestive neoplasm. Dig Liver Dis. 2016;48(3):327-32. doi: http://doi.org/10.1016/j. dld.2015.11.025

20. Kouchaki B, Janbabai G, Alipour A, et al. Randomized double-blind clinical trial of combined treatment with megestrol acetate plus celecoxib versus megestrol acetate alone in cachexia-anorexia syndrome induced by GI cancers. Support Care Cancer. 2018;26(7):2479-89. doi: https://doi.org/10.1007/s00520-018-4047-y

21. Schönsteiner SS, Mißbach HB, Benner A, et al. A randomized exploratory phase 2 study in patients with chemotherapy-related peripheral neuropathy evaluating whole-body vibration training as adjunct to an integrated program including massage, passive mobilization and physical exercises. Exp Hematol Oncol. 2017;6:5. doi: http://doi.org/10.1186/s40164017-0065-6

22. Schwartzberg L, Roeland E, Andric Z, et al. Phase III safety study of intravenous NEPA: a novel fixed antiemetic combination of fosnetupitant and palonosetron in patients receiving highly emetogenic chemotherapy. Ann Oncol. 2018;29(7):1535-40. doi: http://doi. org/10.1093/annonc/mdy169

23. Song G, He Q, Li F, et al. Thalidomide for prevention of chemotherapy-induced nausea and vomiting following highly emetogenic chemotherapy. Australas Med J. 2017;10(3):192-8. doi: https://doi.org/10.21767/ AMJ.2016.2826

24. Tanaka Y, Ueno T, Yoshida N, et al. The effect of an elemental diet on oral mucositis of esophageal cancer patients treated with DCF chemotherapy: a multi-enter prospective feasibility study (EPOC study). Esophagus. 2018;15(4):239-48. doi: https://doi.org/10.1007/ s10388-018-0620-1

25. Yeganeh MZ, Vakili M, Shahriari-Ahmadi A, et al. Effect of oral magnesium oxide supplementation on cisplatin-induced hypomagnesemia in cancer patients: a randomized controlled trial. Iran J Public Health. 2016;45(1):54-62.

26. Oun R, Moussa YE, Wheate NJ. The side effects of platinum-based chemotherapy drugs: a review for chemists. Dalton Trans. 2018;47(10):6645-53. doi: http://doi.org/10.1039/c8dt00838h

27. Simão DAS, Murad M, Martins C, et al. Chemotherapyinduced peripheral neuropathy: review for clinical practice. Rev Dor. 2015;16(3):215-20. doi: http://doi. org/10.5935/1806-0013.20150043

28. Martinho AF, Reis NI, Novo NF, et al. Prevalência dos sintomas depressivos em pacientes oncológicos no ambulatório do Conjunto Hospitalar de Sorocaba. Rev Soc Bras Clin Med. 2009;7(5):304-8.

29. Vadiraja HS, Raghavendra RM, Nagarathna R, et al. Effects of a yoga program on cortisol rhythm and mood states in early breast cancer patients undergoing adjuvant radiotherapy: a randomized controlled trial. Integr Cancer Ther. 2009;8(1):37-46. doi: http://doi. org/10.1177/1534735409331456

30. Pulito C, Cristaudo A, Porta C, et al. Oral mucositis: the hidden side of cancer therapy. J Exp Clin Cancer 
Res. 2020;39(1):210-25. doi: https://doi.org/10.1186/ s13046-020-01715-7

31. Boligon CS, Huth A. O impacto do uso de glutamina em pacientes com tumores de cabeça e pescoço em tratamento radioterápico e quimioterápico. Rev Bras Cancerol. 2011;57(1):31-8. doi: https://doi. org/10.32635/2176-9745.RBC.2011v57n1.683

32. Shumsky A, Bilan E, Sanz E, et al. Oncoxin nutritional supplement in the management of chemotherapy- and/ or radiotherapy-associated oral mucositis. Mol Clin Oncol. 2019;10(4):463-8. doi: http://doi.org/10.3892/ mco.2019.1809

33. Eltweri AM, Howells LM, Thomas AL, et al. Effects of Omegaven ${ }^{\oplus}$, EPA, DHA and oxaliplatin on oesophageal adenocarcinoma cell lines growth, cytokine and cell signal biomarkers expression. Lipids Health Dis. 2018;17(1):19. doi: http://doi.org/10.1186/s12944018-0664-1

34. Ghoreishi Z, Esfahani A, Djazayeri A, et al. Omega-3 fatty acids are protective Against paclitaxel-induced peripheral neuropathy: a randomized double-blind placebo controlled trial. BMC Cancer. 2012;12:355. doi: https://doi.org/10.1186/1471-2407-12-355

35. Hopkinson JB. Psychosocial impact of cancer cachexia. J Cachexia Sarcopenia Muscle. 2014;5(2):89-94. doi: https://doi.org/10.1007/s13539-014-0142-1

36. Fearon KCH, Glass DJ, Guttridge DC. Cancer cachexia: mediators, signaling, and metabolic pathways. Cell Metab. 2012;16(2):153-66. doi: https://doi. org/10.1016/j.cmet.2012.06.011

37. Evans WJ, Morley JE, Argilés J, et al. Cachexia: a new definition. Clin Nutr. 2008;27(6):793-9. doi: https:// doi.org/10.1016/j.clnu.2008.06.013

38. López AP, Figuls MR, Cuchi GU, et al. Systematic review of megestrol acetate in the treatment of anorexia-cachexia syndrome. J Pain Symptom Manage. 2004;27(4):360-9. doi: http://doi.org/10.1016/j.jpainsymman.2003.09.007

39. Schilsky RL, Anderson T. Hypomagnesemia and renal magnesium wasting in patients receiving cisplatin. Ann Intern Med. 1979;(90)6:929-31. doi: http://doi. org/10.7326/0003-4819-90-6-929

40. Schmoll HJ, Aapro MS, Poli-Bigelli S, et al. Comparison of an aprepitant regimen with a multiple-day ondansetron regimen, both with dexamethasone, for antiemetic efficacy in high-dose cisplatin treatment. Ann Oncol. 2006;17(6):1000-6. doi: http://doi.org/10.1093/ annonc/mdl019

41. Rapoport BL, Chasen MR, Gridelli C, et al. Safety and efficacy of rolapitant for prevention of chemotherapyinduced nausea and vomiting after administration of cisplatin-based highly emetogenic chemotherapy in patients with cancer: two randomised, activecontrolled, double-blind, phase 3 trials. Lancet Oncol. 2015;16(9):1079-89. doi: http://doi.org/10.1016/ S1470-2045(15)00035-2

42. Liu Y, Zhang J, Teng Y, et al. Thalidomide improves prevention of chemotherapy-induced gastrointestinal side effects following a modified FOLFOX7 regimen: results of a prospective randomized crossover study. Tumori. 2009;95(6):691-6. doi: https://doi. org/10.1177/030089160909500609

43. Longo F, Mansueto G, Lapadula V, et al. Palonosetron plus 3-day aprepitant and dexamethasone to prevent nausea and vomiting in patients receiving highly emetogenic chemotherapy. Support Care Cancer. 2011;19(8):1159-64. doi: http://doi.org/10.1007/ s00520-010-0930-x

44. Navari RM, Aapro M. Antiemetic prophylaxis for chemotherapy-induced nausea and vomiting. N Engl J Med. 2016;374(14):1356-67. doi: http://doi. org/10.1056/NEJMra1515442 